\title{
Confiabilidade do uso de modelos digitais tridimensionais como exame auxiliar ao diagnóstico ortodôntico: um estudo piloto
}

\author{
Dauro Douglas Oliveira*, Antônio Carlos de Oliveira Ruellas**, Maria Eugênia de Lima Drummond***,

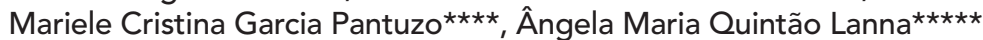

\begin{abstract}
Resumo
Objetivo: os rápidos e contínuos avanços nas Ciências da Computação resultaram no aumento significativo do emprego de novas tecnologias em todos os níveis da sociedade. Na Ortodontia, radiografias e fotografias digitais já são usadas de forma rotineira. A utilização de modelos de estudo digitais vem sendo anunciada como o novo componente da documentação ortodôntica computadorizada. Como acontece quando uma nova tecnologia se torna disponível, o uso de modelos ortodônticos digitais tem gerado controvérsias. Alguns ortodontistas questionam a aplicabilidade de imagens tridimensionais em substituição aos modelos tradicionais de gesso, pois não há na literatura número relevante de estudos que tenham testado adequadamente tal tecnologia. Diante disso, o objetivo desse estudo foi testar a confiabilidade do uso de modelos dentários digitais como exame complementar ao diagnóstico ortodôntico. Metodologia: três examinadores mediram a largura dos dentes permanentes, quatro segmentos dos arcos superiores e inferiores, distâncias intercaninos, distâncias intermolares, trespasses horizontal e vertical em modelos de gesso e em seus correspondentes digitais de seis pacientes, utilizando um paquímetro digital e o programa eModel, respectivamente. Resultados e Conclusões: diante dos resultados, observou-se que todas as medidas avaliadas foram estatisticamente semelhantes nos dois tipos de modelos testados, com exceção das médias encontradas para a largura do dente $45(\mathrm{p}<0,05)$. Entretanto essa diferença é considerada clinicamente aceitável. Comprova-se com esse estudo a confiabilidade do uso dos modelos dentários digitais como exame complementar ao diagnóstico ortodôntico. Além disso, a facilidade de armazenamento de informações, o menor risco de perda de dados durante sua manipulação e transporte, bem como a diminuição do tempo gasto para realizar as medições foram considerados vantagens do uso dessa nova tecnologia na Ortodontia.
\end{abstract}

Palavras-chave: Modelos de estudo. Modelos ortodônticos. Modelos digitais. eModel.

\footnotetext{
Mestre em Ortodontia pela Marquette University, EUA. Doutorando em Ortodontia pela UFRJ. Professor do Mestrado em Ortodontia da PUCMinas - Belo Horizonte-MG

** Mestre e Doutor em Ortodontia pela UFRJ. Professor Adjunto de Ortodontia da UFRJ.

*** Especialista em Ortodontia pela Universidade Vale do Rio Verde - Três Corações-MG.

**** Mestra em Ortodontia pela PUCMinas. Doutoranda em Ciências da Saúde pela UFMG.

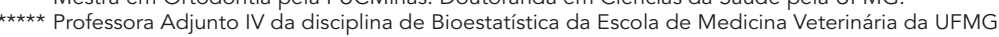




\section{INTRODUÇÃO}

A utilização de modelos dentários é passo indispensável para a obtenção do correto diagnóstico e para a determinação do plano de tratamento ortodôntico mais adequado a cada paciente. A análise de modelos permite avaliar as condições oclusais de cada indivíduo, sem interferência dos tecidos moles da boca, facilitando o estudo do caso. Dessa forma, dados de extrema valia para que o ortodontista melhor compreenda os problemas a serem corrigidos, as dificuldades específicas de cada tratamento e, ainda, as possíveis mecânicas ortodônticas a serem empregadas durante a correção da má oclusão podem ser cuidadosamente estudados ${ }^{5,11}$.

Durante as duas últimas décadas, tem-se verificado, em todos os níveis da sociedade, um desenvolvimento significativo da tecnologia de computação. Na Ortodontia, esses avanços vêm se manifestando, principalmente, em instrumentos de diagnóstico. O emprego de fotografias e radiografias digitais, programas de avaliação cefalométrica e de previsão dos resultados de cirurgias ortognáticas são cada vez mais comuns no cotidiano da clínica ortodôntica atual. Recentemente, a utilização de modelos dentários digitais foi anunciada pela indústria ortodôntica como o novo componente da documentação totalmente digitalizada ${ }^{7,9}$.

Os modelos de estudo tradicionalmente usados na Ortodontia são feitos de gesso e, apesar de imprescindiveis, eles apresentam certas limitações. A necessidade de locais apropriados para sua estocagem, aumentando a demanda por maior espaço físico no consultório e o risco de quebra, o que causaria a destruição permanente do registro do paciente, podem ser considerados desvantagens do emprego de modelos de gesso. Outra limitação do uso desse método de diagnóstico é a dificuldade do estudo de suas informações à distância, principalmente para a Ortodontia contemporânea, onde muitos pacientes requerem abordagem multidisciplinar, por profissionais situados em consultórios diferentes e até mesmo em cidades distintas. Nesses casos, os modelos de gesso têm de ser duplicados e enviados ao outro profissional, aumentando o custo do trabalho e o tempo de espera para troca de informações necessárias ao planejamento ideal do tratamento.

Os modelos dentários digitais foram apresentados como um método que eliminaria as limitações verificadas com os modelos de gesso. Como todo novo exame de diagnóstico, o implemento da tecnologia digital para avaliação de modelos dentários tem gerado controvérsias no meio ortodôntico. A precisão e rapidez para obtenção dos dados de diagnóstico, a facilidade de armazenamento das informações e a possibilidade de se dividir as informações via internet com outros profissionais, em qualquer lugar e a qualquer hora, são apontadas como as principais vantagens dessa nova abordagem de diagnóstico ${ }^{8}$.

Em linhas gerais, existem dois métodos para construção de modelos dentários digitais. O primeiro é conhecido como "escaneamento destrutivo a laser" (laser destructive scanning), processo descrito por Baumrind ${ }^{1}$ como uma análise física destrutiva. Segundo esse autor, o modelo de estudo é revestido em uma matriz sólida e de cor contrastante. Em seguida, a superfície do bloco é cortada paralelamente ao plano oclusal até que o primeiro traço do modelo de gesso se torne aparente. A partir de então, um escaneamento a laser da superfície bidimensional é feito e seus dados são armazenados como uma camada em um arquivo de computador. Posteriormente, outra fatia de 0,003 " do bloco é cortada, um novo escaneamento é feito e suas informações novamente armazenadas. Esse processo é repetido até que toda dentição já irrompida tenha sido mapeada numa série de camadas sucessivas. Conseqüentemente, um mapa tridimensional, consistindo de camadas bidimensionais empilhadas, é obtido. Essa é a metodologia utilizada pela companhia Cadent (Fairview, NJ, EUA) para obtenção de seus modelos digitais.

Segundo Iuorno ${ }^{6}$, o "escaneamento a laser não destrutivo" (laser non-destructive scanning) é o outro método utilizado para a obtenção de um modelo dentário virtual. Esse processo envolve o escaneamento do modelo de gesso como um todo, girando-o nos três planos do espaço, obtendo 
assim, a cópia do modelo original. A acuidade do sensor a laser é de $0,01 \mathrm{~mm}$, o que resulta em um modelo digital com precisão da superfície de aproximadamente $0,01 \mathrm{~mm}$, formado por um emaranhado de aproximadamente 30.000 polígonos por arco dentário. Esse é o método empregado pela empresa Geodigm (Chanhassen, MN, EUA) para a construção de seus modelos digitais. O programa fornecido por essa firma para o processamento das imagens computadorizadas é conhecido como eModel e possui ferramentas que permitem a obtenção das medidas rotineiramente avaliadas nas análises de modelos ortodônticos.

O "sistema OrthoCad" vem sendo testado por alguns pesquisadores. Tomassetti et al. ${ }^{13}$ encontraram diferenças quando compararam quatro métodos para medição da Análise de Bolton (um manual, dois computadorizados e um misto) e concluíram que apesar dessas diferenças não serem estatisticamente significantes, algumas delas podem ter relevância clínica. Eles ainda sugeriram a necessidade de estudos subseqüentes para determinar qual desses métodos é realmente mais preciso e se novas versões dos programas para avaliação de modelos digitais representariam avanços em sua performance.

Garino e Garino ${ }^{4}$ encontraram diferenças estatisticamente significantes entre as medidas obtidas com os modelos de gesso e digital, mas citaram vantagens do programa Orthocad, como a possibilidade de aproximar e girar as imagens tridimensionais do modelo dentário, sem alteração de suas dimensões, além da maior resolução obtida com esse programa.

Zilberman et al. ${ }^{14}$ e Quimby et al. ${ }^{10}$, comparando modelos de gesso e digitais (OrthoCad), mostraram que os dois métodos avaliados são válidos e podem ser reproduzíveis para medidas de tamanho de dentes e larguras dos arcos. Quimby et al. ${ }^{10}$ ainda sugeriram que a facilidade de armazenamento e manipulação dos modelos e o menor tempo necessário à medição dos mesmos são características que podem tornar tal método atrativo para os ortodontistas.

Para que o uso de modelos digitais se torne mais confiável e rotineiro no cotidiano ortodôntico, algumas questões precisam ser mais bem avaliadas. As medidas feitas no modelo digital, projetado na tela do computador, são compatíveis com as aferições realizadas manualmente no modelo de gesso? As análises de modelos, tais como a análise de Bolton ${ }^{3}$ e a de Tanaka e Jonhston ${ }^{12}$, disponíveis nos programas que gerenciam as imagens dos modelos tridimensionais, são confiáveis? Esses parâmetros podem ser repetidos seguidamente e com a mesma precisão? Os programas de computador mais comumente usados para capturar e avaliar imagens de modelos dentários digitais apresentam o mesmo nível de precisão? Dessa forma, esse estudo teve como objetivo testar a confiabilidade do uso dos modelos dentários digitais como exame complementar ao diagnóstico ortodôntico, avaliando possíveis vantagens e desvantagens do uso dessa nova tecnologia na Ortodontia.

\section{MATERIAL E MÉTODOS Obtenção da amostra}

Seis pares de modelos de gesso de pacientes ortodônticos, obtidos antes do tratamento, foram selecionados na Clínica da Disciplina de Ortodontia da Universidade Federal do Rio de Janeiro, obedecendo aos seguintes critérios:

a) Presença de dentadura permanente de primeiro molar esquerdo a primeiro molar direito, tanto na maxila quanto na mandíbula.

b) Ausência de perda de material dentário (tanto no sentido mesiodistal, quanto no sentido ocluso-gengival) por lesão cariosa, fraturas, desgaste interproximal, más formações congênitas ou falhas de moldagem.

c) Modelos de estudo em bom estado de conservação.

Os modelos selecionados foram duplicados, para que as informações relativas aos pacientes não fossem perdidas, e suas cópias foram mantidas na Disciplina de Ortodontia da UFRJ. Os modelos originais foram acondicionados cuidadosamente, para diminuir o risco de quebra durante seu transporte, 
e enviados via correio expresso para a empresa Geodigm Corporation (Chanhassen, MN, EUA). Essa companhia fez o "escaneamento não destrutivo" dos modelos de gesso, para obtenção dos modelos digitais correspondentes. Em seguida, os modelos digitais foram disponibilizados para pesquisa por meio de seu download via internet. A última versão do programa eModel foi fornecida pela companhia e posteriormente utilizada para a obtenção das medidas a serem comparadas com os resultados obtidos com a avaliação manual dos modelos de gesso.

\section{Medidas avaliadas}

Para se comparar a acuidade das medidas obtidas com os modelos dentários de gesso e digitais, os seguintes parâmetros foram aferidos:

a) largura dos dentes;

b) quatro segmentos do arco superior:

- mesial de primeiro molar direito a mesial do canino direito (SSPD);

- mesial do canino direito a mesial do incisivo central direito (SSAD);

- mesial do incisivo central esquerdo a mesial do canino esquerdo (SSAE);

- mesial do canino esquerdo a mesial do primeiro molar (SSPE);

c) quatro segmentos do arco inferior:

- mesial de primeiro molar direito a mesial do canino direito (SIPD);

- mesial do canino direito a mesial do incisi-

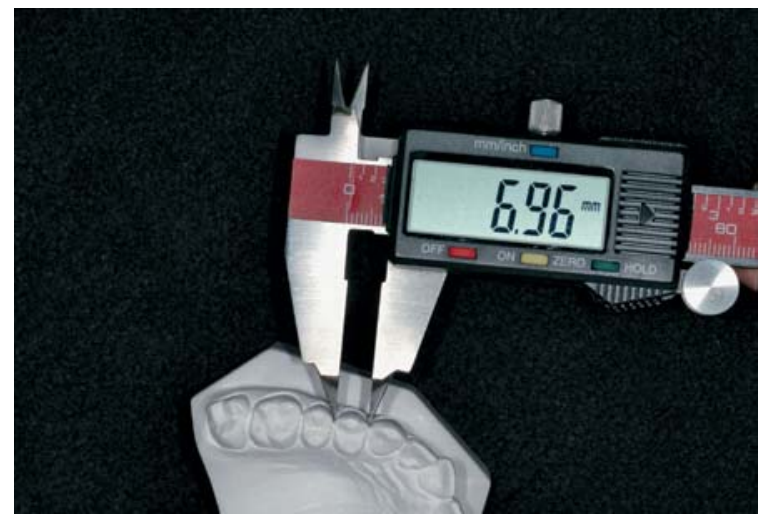

FIGURA 1 - Medida da largura dos dentes com paquímetro digital. vo central direito (SIAD);

- mesial do incisivo central esquerdo a mesial do canino esquerdo (SIAE);

- mesial do canino esquerdo a mesial do primeiro molar (SIPE);

d) distâncias intercaninos superior (DIMS) e inferior (DIMI);

e) distâncias intermolares superior (DIMS) e inferior (DIMI);

f) trespasse horizontal (TH);

g) trespasse vertical (TV).

As medidas no modelo de gesso foram realizadas com um paquímetro digital, calibrado para centésimos de milímetro, de acordo com os seguintes critérios:

a) largura dos dentes: o maior diâmetro no sentido mesiodistal foi medido com as pontas do paquímetro sendo colocadas pela superfície vestibular e perpendicularmente ao longo eixo do dente (Fig. 1);

b) segmentos de arco: os arcos dentários foram divididos em 4 segmentos e medidos com as pontas do paquímetro posicionadas perpendicularmente à superfície vestibular do osso alveolar;

c) distância intercaninos: foi avaliado o segmento de reta medido entre as pontas das cúspides dos caninos direito e esquerdo, de acordo com Bishara et al. ${ }^{2}$;

d) distância intermolares: foi avaliada a maior distância entre as cúspides mesio vestibulares dos

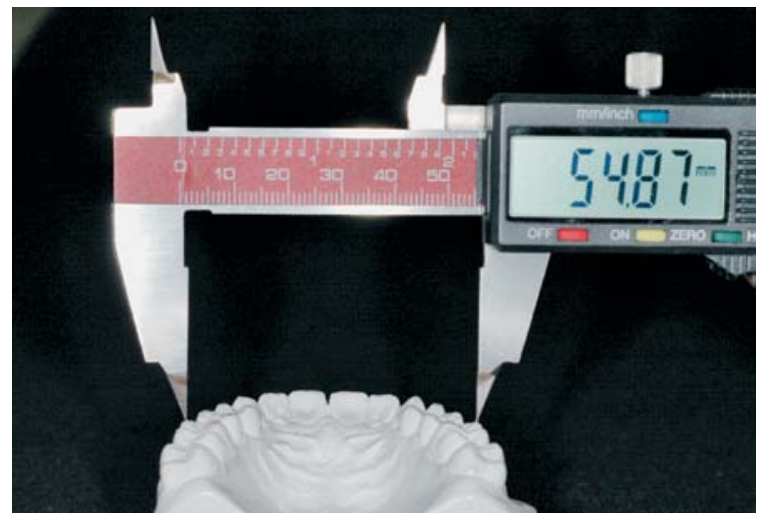

FIGURA 2 - Medida da distância intermolares no modelo de gesso. 
primeiros molares permanentes, também como descrito por Bishara et al. ${ }^{2}$ (Fig. 2);

e) trespasse horizontal: com os modelos em oclusão cêntrica foi feito um ponto com lapiseira $(0,3 \mathrm{~mm}$ de espessura) na margem incisal do incisivo superior mais vestibularizado. Uma régua de madeira sem medições pré-determinadas foi posicionada paralelamente ao plano oclusal, tocando na superfície vestibular do incisivo inferior mais proeminente. Desta forma, o ponto feito na margem do incisivo superior foi transferido para a régua de madeira. $\mathrm{O}$ valor do trespasse horizontal foi obtido medindo-se a distância entre o fim da régua (correspondente à superfície mais vestibularizada dos incisivos inferiores) até o ponto marcado (correspondente ao incisivo superior);

f) trespasse vertical: uma lapiseira $(0,3 \mathrm{~mm}$ de espessura) foi utilizada para registrar no incisivo inferior o ponto correspondente à região de trespasse vertical máximo do incisivo superior. A distância entre esse ponto e a margem incisal do incisivo inferior foi considerada a medida do trespasse vertical.

As aferições dos modelos digitais foram realizadas seguindo as orientações contidas no próprio programa eModel. Antes das medições, os operadores se familiarizaram com a manipulação de tal programa, utilizando o tutorial desse software, que explica os passos necessários para a correta utilização do mesmo. As figuras 3, 4 e 5 ilustram como algumas dessas medições foram realizadas.

Todas as medidas dos dois tipos de modelos foram realizadas por três ortodontistas, sendo repetidas três vezes, com um intervalo de tempo de uma semana, a fim de se avaliar a concordância intra e inter-examinador, além da determinação da reprodutibilidade das medidas. Além disso, os examinadores registraram o tempo necessário para as medições de cada modelo avaliado, com o auxílio de um cronômetro digital.

\section{Análise estatística}

Análises estatísticas foram aplicadas para investigar a confiabilidade das medidas encontradas pelas diferentes técnicas de medição de modelos. O teste de Lilliefors foi realizado para avaliar a distribuição de probabilidade da resposta e os testes de Cochran e Bartlett foram aplicados para verificar possiveis falhas entre examinadores. Finalmente, para a comparação das médias entre os grupos experimentais foi realizado o teste $t$ de Student $(\mathrm{p}<0,05)$.

\section{RESULTADOS E DISCUSSÃO}

A opção pelo "escaneamento não destrutivo" dos modelos de gesso para obtenção dos modelos digitais correspondentes, conhecido como "Sistema eModel", foi feita em função do número reduzido de trabalhos encontrados na literatura que utilizavam esse programa, quando comparado com o escaneamento destrutivo.

As medidas de cada variável foram realizadas pelos três profissionais especializados, tanto nos modelos de gesso quanto nos digitais. De acordo com o teste de Lilliefors, as medidas encontradas se apresentaram harmoniosas e com distribuição seguindo o modelo da curva normal. Os testes de Cochran e Bartlett mostraram que não há valores discrepantes entre os examinadores, revelando que houve boa concordância entre eles, e conseqüentemente não houve diferenças intra-examinadores significantes. A precisão experimental foi alta, conforme se verifica por meio do coeficiente de variação, onde se encontrou valor bem inferior a 30\%, indicando eficiência experimental adequada.

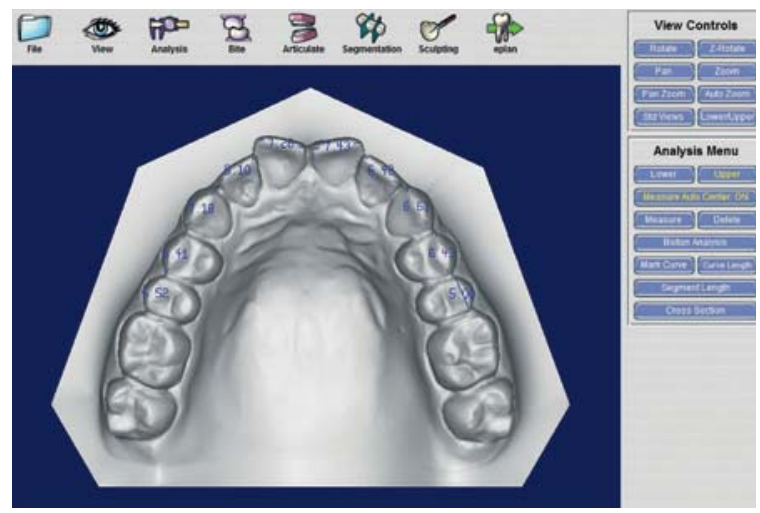

FIGURA 3 - Medidas digitais da largura dos dentes - Programa eModel. 


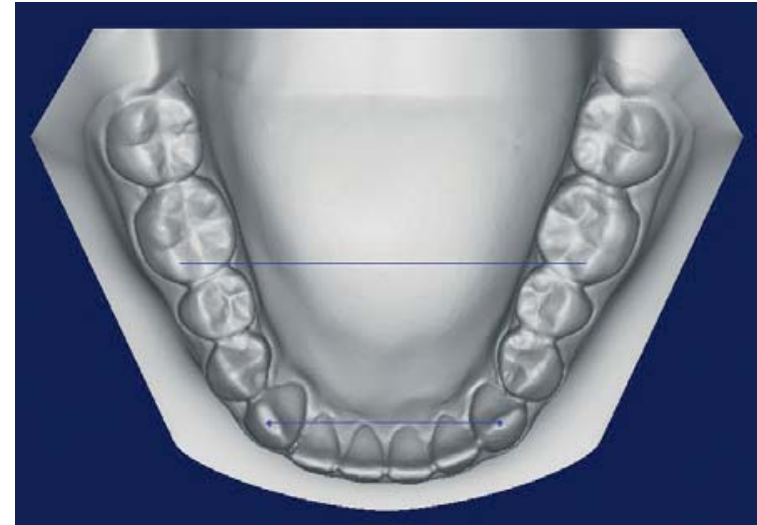

FIGURA 4 - Medida das distâncias intercaninos e intermolares no modelo digital.

O teste $t$ de Student foi utilizado usando amostras pareadas, com o objetivo de verificar se essas medidas apresentavam diferenças significantes. $\mathrm{Na}$ tabela 1 observam-se as comparações entre as médias do comprimento mesiodistal dos dentes em ambos modelos, digital e de gesso. Já as medidas correspondentes aos segmentos avaliados estão agrupadas na tabela 2 , sendo que, em ambas as tabelas, as médias seguidas de letras distintas diferem entre si, com um nível de significância de 5\%.

Para realizar a análise estatística dos resultados, construiu-se um intervalo de confiança de 95\% usando a variável $t$ de Student. Observou-se que todas as medidas avaliadas foram estatisticamente semelhantes nos dois tipos de modelos estudados, exceção feita em relação às médias encontradas para a largura do dente 45 , entretanto essa diferença parece ser clinicamente aceitável. Notou-se ainda que tal diferença foi observada com uma taxa de erro de $5 \%$. Caso a taxa de erro utilizada fosse de $1 \%$, essa diferença não existiria e, assim, todas as médias seriam estatisticamente semelhantes quando os dois tipos de modelos fossem empregados. Os estudos de Zilberman et al. ${ }^{14}$ e Quimby et al. ${ }^{10}$ encontraram resultados semelhantes a esse trabalho, revelando a reprodutibilidade e a confiabilidade das medidas utilizando os modelos digitais. Por outro lado, os achados relatados por Tomassetti et al. ${ }^{13}$, Garino e Garino ${ }^{4}$ são discordantes, pois esses autores encontraram diferenças de relevância clínica, quando compararam

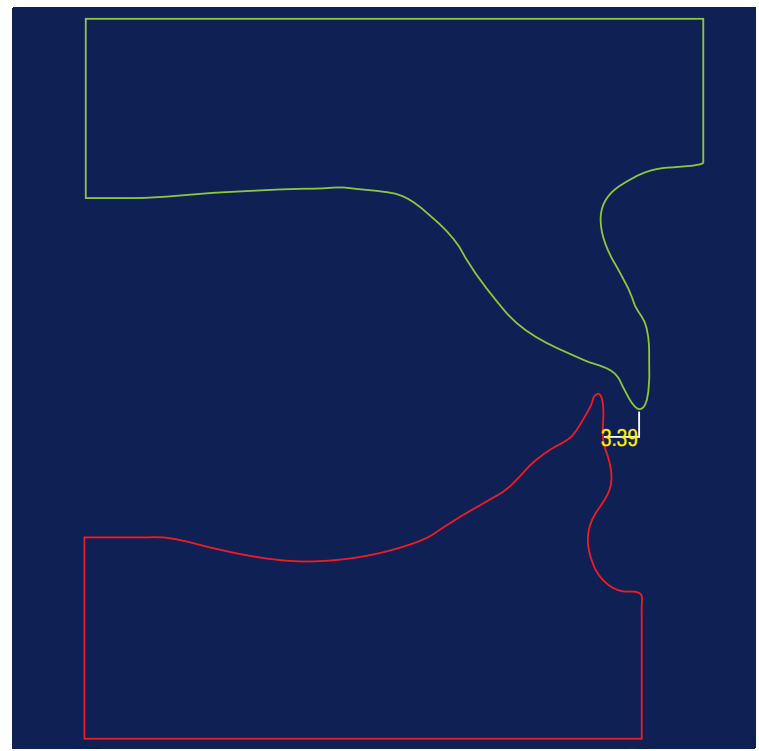

FIGURA 5 - Medida do overjet (vista sagital dos modelos digitais).

os dois tipos de modelos de diagnóstico.

É provável que, dependendo do tipo de medida, os resultados obtidos com o método físico e digital possam ser justificadamente diferentes. Isso poderia estar relacionado à dificuldade na demarcação dos pontos, ou mesmo pela diferença inerente das técnicas de medição utilizadas nesse estudo. Por exemplo, para avaliar a largura dos dentes nos modelos de gesso foi medido o maior diâmetro no sentido mesiodistal, com as pontas do paquímetro sendo colocadas pela superfície vestibular e perpendicularmente ao longo eixo do dente. Entretanto, essas mesmas medidas nos modelos digitais só podem ser realizadas pela vista oclusal dos dentes, o que poderia acarretar alguma diferença nos pontos que foram utilizados na demarcão de tais medidas.

Outro fator que poderia interferir nos resultados está relacionado às diferentes possibilidades de manuseio dos modelos testados. Enquanto os de gesso podem ser manuseados e avaliados em qualquer plano, os digitais, apesar de poderem ser observados em qualquer posição dos três planos do espaço, têm necessariamente de estar numa posição estática para que os pontos de interesse sejam demarcados e as medidas aferidas. 
Tabela 1 - Comparação das médias dos dentes avaliados.

\begin{tabular}{|c|c|c|c|c|}
\hline dente & modelo & média & d.p. & CV (\%) \\
\hline \multirow{2}{*}{15} & gesso & 6,39 a & $\pm 0,31$ & \multirow{2}{*}{5,55} \\
\hline & digital & 6,19 a & $\pm 0,48$ & \\
\hline \multirow{2}{*}{14} & gesso & 6,89 a & $\pm 0,52$ & \multirow{2}{*}{7,97} \\
\hline & digital & 6,74 a & $\pm 0,62$ & \\
\hline \multirow{2}{*}{13} & gesso & $7,42 \mathrm{a}$ & $\pm 0,42$ & \multirow{2}{*}{4,84} \\
\hline & digital & $7,29 a$ & $\pm 0,38$ & \\
\hline \multirow{2}{*}{12} & gesso & $6,22 \mathrm{a}$ & $\pm 0,63$ & \multirow{2}{*}{10,38} \\
\hline & digital & $6,16 \mathrm{a}$ & $\pm 0,71$ & \\
\hline \multirow{2}{*}{11} & gesso & $8,02 \mathrm{a}$ & $\pm 0,62$ & \multirow{2}{*}{6,90} \\
\hline & digital & $7,76 \mathrm{a}$ & $\pm 0,66$ & \\
\hline \multirow{2}{*}{21} & gesso & $7,94 \mathrm{a}$ & $\pm 0,51$ & \multirow{2}{*}{6,26} \\
\hline & digital & $7,72 \mathrm{a}$ & $\pm 0,59$ & \\
\hline \multirow{2}{*}{22} & gesso & $6,40 \mathrm{a}$ & $\pm 0,63$ & \multirow{2}{*}{10,40} \\
\hline & digital & 6,13 a & $\pm 0,78$ & \\
\hline \multirow{2}{*}{23} & gesso & $7,45 \mathrm{a}$ & $\pm 0,48$ & \multirow{2}{*}{5,78} \\
\hline & digital & $7,30 \mathrm{a}$ & $\pm 0,48$ & \\
\hline \multirow{2}{*}{24} & gesso & $6,75 \mathrm{a}$ & $\pm 0,44$ & \multirow{2}{*}{6,67} \\
\hline & digital & 6,57 a & $\pm 0,52$ & \\
\hline \multirow{2}{*}{25} & gesso & 6,34 a & $\pm 0,43$ & \multirow{2}{*}{7,94} \\
\hline & digital & 6,08 a & $\pm 0,58$ & \\
\hline \multirow{2}{*}{35} & gesso & 6,57 a & $\pm 0,36$ & \multirow{2}{*}{5,42} \\
\hline & digital & 6,34 a & $\pm 0,57$ & \\
\hline \multirow{2}{*}{34} & gesso & $6,80 \mathrm{a}$ & $\pm 0,40$ & \multirow{2}{*}{5,08} \\
\hline & digital & 6,65 a & $\pm 0,45$ & \\
\hline \multirow{2}{*}{33} & gesso & 6,57 a & $\pm 0,45$ & \multirow{2}{*}{6,54} \\
\hline & digital & 6,54 a & $\pm 0,47$ & \\
\hline 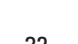 & gesso & 5,27 a & $\pm 0,44$ & 720 \\
\hline 32 & digital & $5,25 a$ & $\pm 0,52$ & $1, \angle 0$ \\
\hline 31 & gesso & $4,82 \mathrm{a}$ & $\pm 0,32$ & 523 \\
\hline & digital & 4,87 a & $\pm 0,37$ & 0,00 \\
\hline & gesso & $4,82 \mathrm{a}$ & $\pm 0,32$ & \\
\hline 41 & digital & 4,91 a & $\pm 0,38$ & $5,4 y$ \\
\hline กา & gesso & 5,26 a & $\pm 0,44$ & 800 \\
\hline 42 & digital & $5,19 a$ & $\pm 0,48$ & 0,90 \\
\hline & gesso & $6,55 \mathrm{a}$ & $\pm 0,51$ & \\
\hline 40 & digital & $6,56 \mathrm{a}$ & $\pm 0,51$ & 1,09 \\
\hline 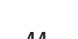 & gesso & 6,83 a & $\pm 0,39$ & 5? \\
\hline 44 & digital & 6,71 a & $\pm 0,53$ & J, \\
\hline 45 & gesso & 6,52 a & $\pm 0,37$ & 192 \\
\hline & digital & $6,31 \mathrm{~b}$ & $\pm 0,53$ & \\
\hline
\end{tabular}

*Médias seguidas de letras distintas por região diferem entre si pelo teste t de Student $(p<0,05)$. 
Tabela 2 - Comparação das médias dos segmentos avaliados.

\begin{tabular}{|c|c|c|c|c|}
\hline segmento & modelo & média & d.p. & CV (\%) \\
\hline \multirow{2}{*}{ SSPD } & gesso & $21,81 \mathrm{a}$ & $\pm 0,71$ & \multirow{2}{*}{3,79} \\
\hline & digital & 21,97 a & $\pm 0,91$ & \\
\hline \multirow{2}{*}{ SSAD } & gesso & 15,24 a & $\pm 0,87$ & \multirow{2}{*}{6,32} \\
\hline & digital & 15,49 a & $\pm 1,10$ & \\
\hline \multirow{2}{*}{ SSAE } & gesso & 15,48 a & $\pm 1,18$ & \multirow{2}{*}{7,50} \\
\hline & digital & 15,60 a & $\pm 1,08$ & \\
\hline \multirow{2}{*}{ SSPE } & gesso & 21,83 a & $\pm 0,79$ & \multirow{2}{*}{4,15} \\
\hline & digital & $22,09 \mathrm{a}$ & $\pm 0,99$ & \\
\hline \multirow{2}{*}{ SIPE } & gesso & 21,31 a & $\pm 0,77$ & \multirow{2}{*}{3,78} \\
\hline & digital & 21,33 a & $\pm 0,83$ & \\
\hline \multirow{2}{*}{ SIAE } & gesso & $11,19 \mathrm{a}$ & $\pm 0,85$ & \multirow{2}{*}{7,38} \\
\hline & digital & $11,27 \mathrm{a}$ & $\pm 0,77$ & \\
\hline \multirow{2}{*}{ SIAD } & gesso & 10,98 a & $\pm 0,49$ & \multirow{2}{*}{4,54} \\
\hline & digital & $11,00 \mathrm{a}$ & $\pm 0,51$ & \\
\hline \multirow{2}{*}{ SIPD } & gesso & $20,91 \mathrm{a}$ & $\pm 1,16$ & \multirow{2}{*}{4,71} \\
\hline & digital & $21,26 \mathrm{a}$ & $\pm 0,73$ & \\
\hline \multirow{2}{*}{ DIMIS } & gesso & 50,28 a & $\pm 3,47$ & \multirow{2}{*}{7,27} \\
\hline & digital & 50,06 a & $\pm 3,63$ & \\
\hline \multirow{2}{*}{ DICS } & gesso & 33,54 a & $\pm 2,16$ & \multirow{2}{*}{6,49} \\
\hline & digital & 33,30 a & $\pm 2,11$ & \\
\hline \multirow{2}{*}{ DIMI } & gesso & $44,21 \mathrm{a}$ & $\pm 2,90$ & \multirow{2}{*}{6,80} \\
\hline & digital & 44,6 a & $\pm 3,54$ & \\
\hline \multirow{2}{*}{ DICI } & gesso & $25,20 \mathrm{a}$ & $\pm 2,34$ & \multirow{2}{*}{9,33} \\
\hline & digital & 25,33 a & $\pm 2,23$ & \\
\hline \multirow{2}{*}{ TV } & gesso & $4,10 \mathrm{a}$ & $\pm 1,44$ & \multirow{2}{*}{38,28} \\
\hline & digital & 3,88 a & $\pm 1,53$ & \\
\hline \multirow{2}{*}{ TH } & gesso & 4,34 a & $\pm 1,13$ & \multirow{2}{*}{28,98} \\
\hline & digital & $4,26 \mathrm{a}$ & $+1,28$ & \\
\hline
\end{tabular}

*Médias seguidas de letras distintas por região diferem entre si pelo teste t de Student $(p<0,05)$.

Durante o desenvolvimento do presente estudo, observou-se que o tempo necessário para medir os modelos digitais foi em média $40 \%$ menor que o tempo gasto nas medições dos modelos de gesso. Essa observação evidencia uma maior agilidade quando utilizamos os modelos digitais para auxílio de diagnóstico ortodôntico. Esse fato também foi relatado por Zilberman et al. ${ }^{14}$ em seus experimentos.

Outras vantagens da utilização de modelos digitais que puderam ser observadas com o desenvolver desse estudo dizem respeito, primeiramente, à facilidade de sua manipulação após bre- ve familiarização com o programa. Em segundo, o fácil armazenamento das informações, eliminando a necessidade de se ter espaços físicos grandiosos para estocagem dos modelos de gesso, o que a cada dia mais se torna um problema na rotina dos consultórios ortodônticos, principalmente nas grandes regiões metropolitanas. Por último, a comunicação entre os profissionais da área é facilitada, visto que os modelos podem ser enviados pela internet, evitando possíveis quebras durante o transporte e manuseio dos modelos físicos, vantagem essa, também citada por Marcel $^{8}$. 
A substituição de métodos de diagnóstico convencionais por outros tecnologicamente mais avançados tem sido uma constante na área médico-odontológica nos últimos anos. A utilização de modelos de estudos digitais parece ter potencial para se acrescentar a essa tendência, o que poderia significar um desenvolvimento no processo de armazenamento das informações necessárias ao diagnóstico ortodôntico. Entretanto, o alto custo para a obtenção dos modelos digitais dificulta sua inclusão na rotina clínica do ortodontista brasileiro, visto que esse exame complementar é recente e o domínio de sua tecnologia pertence a empresas norte-americanas. Mesmo assim, questiona-se se a comprovada acuidade das medidas obtidas com esse método e suas outras vantagens aqui citadas não justificariam investimentos para o desenvolvimento de tecnologias nacionais nessa área?

\section{CONCLUSÕES}

1) Os modelos ortodônticos digitais se mostraram tão confiáveis quanto os de gesso para afe- rição de medidas comumente utilizadas para o diagnóstico, uma vez que não houve diferenças estatisticamente significantes.

2) A única medida com diferenças estatisticamente significantes foi o comprimento mesiodistal do dente 45. Entretanto, tais valores se mostraram dentro de padrões clinicamente aceitáveis.

3) $\mathrm{O}$ armazenamento e a manipulação facilitados, com menor risco de perda de informações, a possibilidade de estudo dos modelos à distância e o menor tempo necessário para medições são outros pontos positivos no emprego dos modelos digitais na Ortodontia.

4) $O$ potencial para utilização rotineira dessa nova tecnologia é inegável. Entretanto, seu alto custo para os padrões brasileiros pode retardar esse acontecimento, sendo interessante avaliar-se a possibilidade de investimentos para o desenvolvimento de tecnologia nacional na área.

\title{
Reliability of three-dimensional digital casts as a diagnostic tool for orthodontic treatment planning: a pilot study
}

\begin{abstract}
Aim: the fast and continuous advances in computer sciences have resulted in an increased usage of new technologies in all levels of the modern society. Orthodontics has also been influenced by this phenomenon. Digital radiographs and photographs have been commonly used in Orthodontics offices. Recently, digital study models have been advertised as the latest component of fully digitized orthodontic records. When a new diagnostic technology becomes available it may initially generate controversy, and with digital orthodontic casts it has not been different. Some orthodontists may question the reliability of this new diagnostic tool since there is not enough literature to support the substitution from traditional stone casts to digital models. Therefore, the aim of the present study was to compare the reliability of digital orthodontic models and stone casts as a diagnostic aid. Methods: three examiners measured the width of the permanent teeth, intercanine and intermolar distances, overbite and overjet of stone and the corresponding digital casts from six patients. A digital caliper was used for measuring the stone casts and the eModel software to evaluate the digital models. Results and Conclusions: the results of this study showed no statistically significant difference among any of the measurements tested, except by the width of the lower second right premolar $(p<0.05)$. However, these differences were not considered clinically relevant. These findings show that digital orthodontic casts are as reliable as stone study models as a diagnostic tool for orthodontic treatment planning. The easy data storage, the lower risk of breakage during its handling and transportation, and also the shorter time needed to obtain the diagnostic information were considered additional advantages of this new technology.
\end{abstract}

Key words: Study models. Orthodontic models. Digital casts. eModel. 


\section{REFERÊNCIAS}

1. BAUMRIND, S. Introduction. Semin Orthod, Philadelphia, v. 7, no. 4, p. 222, 2001

2. BISHARA, S. E. JAKOBSEN, J R. TREDER, J.NOWAK, A. Arch width changes from 6 weeks to 45 years of age. Am J Orthod Dentofacial Orthop, St. Louis, v. 111, no. 4, p. 401-409, 1997.

3. BOLTON, W. A. Disharmony in tooth size and its relation to the analysis and treatment of malocclusion. Angle Orthod, Appleton, v. 28 , no. 3, p. 113-130, 1958

4. GARINO, F.; GARINO, G. B. Comparison of dental arch measurements between stone and digital casts. World J Orthod, Carol Stream, v. 3, p. 250-254, 2002

5. HOWES, A. E. Arch width in the premolar region - still the major problem in Orthodontics. Am J Orthod, St. Louis, v. 43, p. 5-31, 1957.

6. IUORNO JR., F. The third dimension. Orthod Prod, [s.I.], v. 9 p. 14-16, 2002

7. KURODA, T: MOTOHASHI, N.: TOMINAGA, R.; IWATA, K Three-dimensional cast analyzing system using laser scanning. Am J Orthod Dentofacial Orthop, St. Louis, v. 110, no. 4, p. 365-369, 1966.

8. MARCEL, T. Three-dimensional on-screen virtual models. Am Orthod Dentofacial Orthop, St. Louis, v. 119, no. 6, p. 666668, 2001.
9. MOTOHASHI, N.; KURODA, T. A 3D computer-aided design system applied to diagnosis and treatment planning in Orthodontics and Orthognathic Surgery. Eur J Orthod, Oxford, v. 21, no. 3, p. 263-274, 1999.

10. QUIMBY, M. L.; VIG, K. W. L.; RASHID, R. G.; FIRESTONE, A. $R$. The accuracy and reliability of measurements made on computer-based digital models. Angle Orthod, Appleton, v. 74, no. 3, p. 298-303, 2004.

11. REDMOND, W. R. Digital models: a new diagnostic tool. J Clin Orthod, Boulder, v. 35, no. 6, p. 386-387, 2001.

12. TANAKA, M. M.; JOHNSTON, L. E. The prediction of the size of unerupted canines and premolars in a contemporary orthodontic population. J Am Dent Assoc, Chicago, v. 88, p. 798-801, 1974.

13. TOMASETTI, J. J.; TALOUMIS, L. J.; DENNY, J. M.; FISHER JR. A comparison of 3 computerized Bolton tooth-size analyses with a commonly used method. Angle Orthod, Appleton, v. 71 no. 5, p. 351-357, 2001.

14. ZILBERMAN, O.; HUGGARE, J. A. V.; PARIKAKIS, K. A. Evaluation of the validity of tooth size and arch width measurements using conventional and three-dimensional virtual orthodontic models. Angle Orthod, Appleton, v. 73, no. 3, p. 301-306, 2003.

\section{Endereço de correspondência}

Dauro Douglas Oliveira

Av. Cristóvão Colombo, 550/404, Savassi

CEP: 30.140-150 - Belo Horizonte/MG

E-mail: dauro.bhe@terra.com.br 